\title{
A Multiplicidade do TDAH nas Diferentes Versões Produzidas pelas Ciências no Brasil
}

\author{
Rodrigo Ramires Ferreira* (D) \& Murilo dos Santos Moscheta (D) \\ Universidade Estadual de Maringá, Maringá, PR, Brasil
}

\begin{abstract}
RESUMO - A partir da Teoria Ator-Rede, esta revisão teve como objetivos discutir as diferentes realidades performadas pela literatura científica brasileira sobre o TDAH e descrever a produção de versões acerca do fenômeno por meio do funcionamento de suas redes heterogêneas. Selecionamos 80 artigos científicos brasileiros das bases de dados Lilacs, Pepsic, BVS, Scielo e IndexPsi utilizando o descritor TDAH. A discussão aponta para diferentes versões do TDAH, que se distanciam, se conectam, se sobrepõem e agenciam diferentes realidades por meio das relações entre agentes humanos e não humanos que participam da construção destas versões. Espera-se que a presente pesquisa possa contribuir com um olhar para o TDAH por meio de seus agenciamentos, com base na busca de uma compreensão mais ampla e híbrida deste fenômeno.
\end{abstract}

PALAVRAS-CHAVE: TDAH, Teoria Ator-Rede, revisão de literatura

\section{The Multiplicity of ADHD in Different Versions Produced by Science in Brazil}

\begin{abstract}
From an Actor-Network Theory standpoint, this research aimed at discussing the different ADHD realities enacted by Brazilian scientific literature and to describe the production of different versions about the phenomenon by the action of its heterogenic networks. We selected 80 Brazilian scientific papers from Lilacs, Pepsic, BVS, Scielo and IndexPsi databases using ADHD as a keyword according to DeCS (health science descriptors). The discussion indicates three different versions of ADHD that connect, detach, overlap and activate different realities by agency of humans and non-humans actors. We hope this research can contribute to creating an approach to ADHD that considers its assemblage and that foster a broad and hybrid comprehension of this phenomenon.
\end{abstract}

KEYWORDS: ADHD, Actor-Network Theory, literature review

O Transtorno de Déficit de Atenção e Hiperatividade (TDAH) é um fenômeno atravessado por inúmeras controvérsias, discursos e materialidades que tem ganhado crescente atenção das produções científicas ao longo do século XX e XXI. É também nesta época que se proliferam os discursos que problematizam a ciência enquanto produção de um saber neutro, objetivo e generalizável. As várias formas de se falar do TDAH, os discursos e as práticas que delas surgem serão retomados aqui em um sentido no qual os produtos da ciência não são produtos naturais, mas efeitos de movimentos, práticas e agenciamentos que estão situados historicamente.
Nesse sentido, as formas como se performam as práticas e os modos de se falar do TDAH propostos por um modelo hegemônico de ciência trazem grandes prejuízos ao propor intervenções baseadas em um único modelo biologizante, centrado na verdade científica e em um discurso de déficit. $\mathrm{Na}$ trilha das contribuições de Bruno Latour e da Teoria Ator-Rede, devemos olhar para os efeitos dos discursos e das práticas científicas, entendendo a realidade como múltipla. A Teoria Ator-Rede se constitui como um campo heterogêneo de estudos, no qual aquilo que habitualmente é entendido como a realidade e que usualmente é tomado como o objeto de estudo das ciências é tratado como efeito

\footnotetext{
*E-mail: rodrigoramirez1@gmail.com

- Submetido: 26/09/2016; Revisado: 11/03/2018; Aceito: 20/03/2018.
} 
de associações e arranjos de elementos distintos. São circulações, alianças, fluxos e movimentos compostos por atores humanos e não-humanos (chamados de actantes) que redefinem mútuas relações. Segundo Moraes (2004), "uma ciência definida como rede de atores não se caracteriza por sua racionalidade e objetividade ou pela veracidade dos fatos por ela engendrados. Todas essas noções... devem ser entendidas como efeitos" (p. 3).

Para construir uma realidade, as/os cientistas precisam angariar aliados, uma gama de actantes que devem ser mobilizados: política, discursos, laboratórios, instituições, textos científicos, para performar uma "realidade" (Moraes, 2001/2002). Nesse sentido, entendemos que os artigos científicos que falam acerca do TDAH fazem parte desta relação e desta construção, podendo constituir o objeto da própria investigação científica. Segundo Moraes (2001/2002, p. 3-4), "estudar a ciência em ação significa estudar a ciência como um processo de fabricação do mundo - social e natural".

Partimos do pressuposto de que o TDAH não é único, ou seja, não há uma única versão possível de descrevêlo, mas sim múltiplas versões que podem ou não estar relacionadas. Nosso objetivo foi de buscar as dissonâncias ou controvérsias nos estudos disponíveis na literatura científica brasileira e de que forma e que aliados estes buscam para performar realidades acerca do TDAH.

\section{METODOLOGIA}

Elaboramos uma estratégia de revisão sistemática da literatura de modo a visibilizar diferentes versões do TDAH. Guiamo-nos pela questão chave: quais são os discursos acionados ou utilizados pela literatura científica brasileira na construção do sentido sobre TDAH na infância entre os anos 1980 e 2015 ? A busca foi realizada no período de 20 a 30 de janeiro de 2015 nas bases de dados Lilacs, Pepsic, BVS, Scielo e IndexPsi utilizando o descritor TDAH na base de descritores em saúde (DeCS). Após as buscas nas bases de dados, identificamos 885 trabalhos sobre TDAH, publicados entre 1980 e 2015.

Optamos por incluir no estudo somente artigos em língua portuguesa produzidos no Brasil para traçar um entendimento acerca destes na literatura nacional. Justificamos este recorte com base no mais recente levantamento do Sistema Nacional de Gerenciamento de Produtos Controlados (SNGPC), datado de 2013, que aponta um crescimento de $75 \%$ no consumo do metilfenidato no Brasil, medicamento utilizado para tratar o dito transtorno, entre os anos de 2009 e 2011, considerando a faixa etária de 6 a 16 anos (ANVISA, 2013). Ao considerarmos o período de uma década (2001 a 2011) entre todas as faixas etárias, o aumento no consumo chega a $1600 \%$, colocando o Brasil em segundo lugar no ranking mundial de maiores consumidores de metilfenidato, atrás apenas dos EUA (Ferreira, 2015).

Depois de excluídos 314 estudos em língua estrangeira e 252 estudos repetidos, passamos à leitura de títulos, resumos e textos completos para aplicarmos os critérios de inclusão (artigos sobre TDAH na infância na literatura científica brasileira) e os de exclusão (teses, palestras e livros; estudos com adultos; estudos que não tratavam especificamente sobre TDAH; estudos sobre comorbidades; artigos com restrição de acesso ou com visualização parcial; estudos que tratavam especificamente de medicamentos ou efeitos medicamentosos). Chegamos ao número de 80 artigos selecionados para a presente revisão.

Após essa primeira etapa de seleção e organização dos trabalhos, realizamos uma leitura completa dos artigos buscando os principais elementos elencados por seus/ suas respectivos/as autores/as, que nos poderiam mostrar pistas dos agenciamentos humanos e não humanos que contribuíram para a construção das versões sobre TDAH em cada artigo selecionado. Os registros foram feitos em termos dos recursos teóricos e metodológicos utilizados, bem como do modo de cada texto buscar produzir conhecimento acerca do "transtorno".

Outra etapa consistiu na busca pelos metadados dos estudos e incluiu: (1) ano de publicação por meio das bases de dados; (2) estratificação dos periódicos nos quais se encontram publicados os artigos pelos conceitos do Qualis - CAPES; (3) número de citações dos artigos pelos motores de busca Scopus e Google Acadêmico; e (4) área do conhecimento e filiação institucional de autores/as encontradas na Plataforma Lattes.

A etapa final objetivou selecionar três artigos entre os 80 incluídos em nossa pesquisa para ilustrar, contrastar e discutir a construção das diferentes versões sobre o TDAH. A leitura foi realizada com duplo foco: (1) analisamos aquilo que está para além do texto, como o qualis, as bases de dados, filiações disciplinares dos/as autores/as, citações, que sugerem o modo como o texto participa em uma rede de produção e sustentação de uma dada versão; (2) analisamos o conteúdo dos artigos e elaboramos três eixos de análise que dizem respeito aos focos 1 e 2, quais sejam: (a) que aliados buscam ou angariam para tornar fato suas sentenças; (b) como estes falam do TDAH; e (c) que nós de atores humanos e não humanos participam desse processo.

\section{Das Redes dos Artigos I: Um Olhar Geral para Todos os Artigos Selecionados}

Compartilhando a afirmação de John Law (1986) de que o texto é arma secreta da ciência, nesta seção descreveremos as redes dos artigos científicos que selecionamos para este estudo. O texto científico é uma tradução daquilo que "acontece no laboratório", vem carregado com toda a gama 
de elementos envolvidos em seu contexto de produção: pessoas, cadeiras, fórmulas, tabelas, instituições, revistas e máquinas, uma estrutura complexa que constrói um mundo e transcende, em certa medida, o espaço-tempo (Law, 1986).

Nesse sentido, não basta o texto científico ser escrito, deve ter a capacidade de "fazer agir" à distância. Esta capacidade, por sua vez, perpassa por dois argumentos importantes na construção da agência de um texto em particular. Para Law (1986), além da produção do texto em si, que diz respeito ao modo como as informações são construídas e dispostas na escrita, há também negociações que devem ser consideradas em termos de destino do texto científico, como o local (revista) a ser enviado e para quem ou a que será direcionado.

Há, no Brasil, aquilo que a Coordenação de Aperfeiçoamento de Pessoal de Nível Superior (CAPES) ambiciona chamar de termômetro da produção científica: o Qualis. Este é um conjunto de procedimentos que mede a qualidade da produção científica a partir da qualidade do periódico no qual esta produção é publicada. Os estratos indicativos são: A1, A2, B1, B2, B3, B4, B5, e C sendo A1 o mais elevado.

Quanto ao qualis das publicações, observamos que a maioria dos trabalhos encontram-se no extrato B2, com $28,8 \%$ dos artigos, e que apenas $8,8 \%$ estão publicados no extrato de maior "prestígio", o A1. Se considerarmos que os periódicos classificados nos estratos B1 e B2 são publicações que estão em busca ou em processo de ascensão de seu qualis, o número de artigos nesses periódicos tidos como de melhor qualidade é de $80,1 \%$. Podemos considerar que a produção científica sobre TDAH no Brasil tem ocupado um espaço de significância, pois sua discussão se coloca e se apresenta nos principais periódicos.

Para a análise do número de citações dos artigos selecionados, utilizamos o motor de buscas Google Acadêmico, que elenca citações em artigos, teses, dissertações, palestras etc., agregando algumas bases de dados como Scielo e PePsic, bem como o Scopus, que referencia somente artigos em periódicos científicos. A análise do número de citações de um artigo científico é relevante, pois tal como afirma Latour (2011), "o status de uma afirmação depende das afirmações ulteriores. Seu grau de certeza aumenta ou diminui, dependendo da sentença seguinte que a retomar" (p. 40).

Isso significa dizer que quanto maior o número de citações, aliados de um texto, mais fato ele se torna. Contudo, isso não quer dizer que a sentença é uma verdade absoluta. Esse ponto é marcante na literatura científica acerca do TDAH, pois, por exemplo, o artigo com o maior número de citações, 183 nos dois motores de busca, intitulado "Transtorno de déficit de atenção/hiperatividade: atualização", de Rohde e Halpern (2004), aponta o TDAH como um transtorno de base neuroquímica e ambiental, sem causas precisas, mas é aceito pela literatura científica: "Apesar do grande número de estudos já realizados, as causas precisas do TDAH ainda não são conhecidas. Entretanto, a influência de fatores genéticos e ambientais no seu desenvolvimento é amplamente aceita na literatura" (Rohde \& Halpern, 2004, p. 62). Percebemos que a aceitação do TDAH como um transtorno, neste caso, está no número de aliados que esse tipo de sentença possui, pois há um grande número de pesquisas que a afirmam, anteriores à pesquisa de Rohde e Halpern (2004), fazendo com que o texto possa agir à distância como diz Law (1986). Nessa direção, afirmar algo que é impreciso prescinde de muitas afirmações da literatura científica, ou seja, a substância do que se diz está no fato de que foi dito várias vezes por outros, ainda que para além do dito, os autores reconheçam apenas a imprecisão. $\mathrm{O}$ trecho do texto de Rohde e Halpern (2004) mostra como a ciência é feita de recursividade. Essa imprecisão é, em outros textos, mote para a arregimentação de outros recursos, que levam a criação de outros fatos, como um uso pernicioso do diagnóstico e biologização. Um exemplo disso pode ser visto a seguir.

O artigo de Brzozowski e Caponi (2012) intitulado "Determinismo biológico e as neurociências no caso do transtorno de déficit de atenção com hiperatividade" faz uma crítica à etiologia biológica do TDAH. Nas palavras das autoras: "questionamos a ideia de que o TDAH é um transtorno biológico, que pode ser identificado por meio de técnicas de neuroimagem e que é tratável por meio de psicoestimulantes como o metilfenidato" (p. 943). Essa sentença das autoras também arregimenta muitos recursos, recorrendo a pesquisas anteriores que a reafirmam, inclusive o argumento de que as causas (biológicas) precisas do TDAH não são reconhecidas. $\mathrm{O}$ que queremos apontar com esses dois exemplos é que "em suma, a construção de fatos e máquinas é um processo coletivo" (Latour, 2011, p. 43) e passível de ser encenado de diferentes maneiras.

Retomando as associações entre qualis e o número de citações, utilizando os dois artigos citados como exemplo, temos que: o primeiro, que conta com o maior número de citações (183), está publicado no estrato B2, na área de medicina; o segundo artigo do exemplo acima, em estrato B1, na área de saúde coletiva. Curiosamente, o artigo com o maior número de citações publicado em estrato A1 de sua área (psicologia) é apenas o oitavo em números de citações, com 37 no total.

Nesse sentido, se os artigos mais citados não são os mais bem avaliados (em termos de qualis dos periódicos), podemos questionar: (a) o próprio dispositivo qualis como um termômetro que mede a qualidade da produção científica no Brasil. Isso porque encontramos artigos publicados em periódicos que não estão nos melhores estratos de avaliação, mas que, ainda sim, têm grande impacto na literatura científica; (b) esse impacto pode estar mais relacionado com as afirmações e agenciamentos que um artigo produz (e/ou permite produzir e sustentar) do que com os dispositivos instituídos de certificação de qualidade científica. 
As áreas do conhecimento apresentadas na Figura 1 estão ocupadas em falar acerca do TDAH e são permeadas por variados discursos. A psicologia é o exemplo mais claro dessa afirmação. Há 38 artigos de autoria de pessoas da área de psicologia e a multiplicidade de versões sobre o TDAH nesses artigos acompanha a multiplicidade de versões de psicologia e suas subáreas como, por exemplo, clínica, educação, desenvolvimento e psicologia social. $\mathrm{Na}$ área da medicina e suas subáreas, como pediatria, psiquiatria, neurologia, entre outras, observamos esse mesmo movimento.

O TDAH não é único nem matéria de pesquisa de uma única área do conhecimento, a qual detém o poder científico exclusivo de falar sobre o fenômeno, ainda que algumas chamem para si essa premissa nas entrelinhas de seus textos. Talvez um exemplo disso seja o artigo de Gomes et al. (2007) intitulado "Conhecimento sobre o transtorno do déficit de atenção/hiperatividade no Brasil", quando este afirma que "De fato, os estudos têm demonstrado que mesmo as abordagens combinadas, por exemplo, medicação e psicoterapia comportamental, não são eficazes em comparação com o uso isolado de medicamentos" (p. 100), utilizando autores que corroboram essa sentença, isto é, os quais são "aliados". A afirmação do autor chama para as "ciências do biológico" a responsabilidade de tratar o TDAH com medicamentos, que seriam, para essa versão, mais eficazes que outras terapias para tratar o "transtorno". Entre outros agentes para além da filiação com as "ciências do biológico", é interessante apontar que o autor do texto acima trabalha para a Norvatis Biociências, indústria farmacêutica responsável pela fabricação da medicação para tratamento do TDAH. Isto permite interrogar acerca do interesse financeiro que atravessa a construção de determinadas versões sobre TDAH, isto é, as negociações envolvidas na produção do texto que guardam relações com as heterogeneidades dos atores humanos e não humanos envolvidos nas relações (Law 1986), por exemplo, os remédios, o empregador do autor, as máquinas, os financiamentos, os aliados etc.

Temos ainda que o periódico com o maior número de publicações sobre o TDAH é a Revista da Academia Brasileira de Neurologia, a Arquivos de Neuropsiquiatria, classificada no estrato B2 do qualis em medicina II, que engloba, entre outras áreas, neurociência e psiquiatria, com um total de nove artigos nessas áreas. A área com o maior número de artigos (psicologia) publica nos mais variados periódicos e a revista com o maior número de artigos publica apenas textos de uma área (medicina/neuropsiquiatria), um fenômeno na ciência que vai ao encontro do que aponta Law (1986): "o texto só é forte se (a) seus componentes tem força no ponto onde o texto é recebido e (b) são reconhecidos como sendo apropriadamente emprestados e justapostos" (p. 68).

Números, estrato, citações, artigos, qualis, aliados, áreas, autores, revistas e ciência são pontos, nós relacionais, que fazem performar a ciência sobre o TDAH por meio

\begin{tabular}{|ll|}
\multicolumn{1}{c}{ Medicina } \\
\hline Neurologia & 3 \\
Neuropediatria & 2 \\
Pediatria & 1 \\
Psiquiatria & 7 \\
TOTAL & $\mathbf{1 3}$ \\
\hline
\end{tabular}

\begin{tabular}{|ll|}
\multicolumn{2}{c}{ Psicologia } \\
\hline Psicologia & 7 \\
Clínica & 4 \\
Desenvolvimento & 5 \\
Social & 5 \\
Educação & 7 \\
Gestalt & 3 \\
Neurociências & 2 \\
Psicanálise & 3 \\
Psicometria & 1 \\
Psiquiatria & 1 \\
TOTAL & $\mathbf{3 8}$ \\
\hline
\end{tabular}

Terapia Ocupacional

Terapia Ocupacional 1
Biociências

\begin{tabular}{|ll|}
\hline Biociências & 1 \\
Genética & 1 \\
TOTAL & $\mathbf{2}$ \\
\hline
\end{tabular}

Fonoaudiologia

Fonoaudiologia 2

Psicopedagogia 1

Linguística

TOTAL

\section{Estatística}

\begin{tabular}{|c|}
\hline Epidemiologia 1 \\
\hline Neuropsicologia \\
\hline Neuropsicologia 2 \\
\hline
\end{tabular}

Fisioterapia

\begin{tabular}{|ll|}
\hline Fisioterapia & 1 \\
Educação & 1 \\
TOTAL & $\mathbf{2}$ \\
\hline
\end{tabular}

\section{Química}

Educação Especial 1

Farmácia

\begin{tabular}{|ll|}
\hline Farmácia & 1 \\
Saúde Coletiva & 3 \\
TOTAL & $\mathbf{4}$ \\
\hline
\end{tabular}

Educação

Educação 3

Pedagogia

Pedagogia 3

Psicopedagogia

Psicopedagogia 2

Ciências da computação

Neurociências 1

Figura 1. Grande área e subáreas das/os autoras/es e o total de artigos por área.

Nota: As subáreas foram construídas a partir da área de especialização dos/as primeiros/as autores/as. Por isso, por exemplo, Saúde Coletiva aparece como subárea da Farmácia, pois a autora formada em farmácia com pós-graduação em saúde coletiva, ou então educação como subárea da Fonoaudiologia e assim por adiante. 
das publicações no Brasil. O texto científico não age por si só e se é forte o suficiente para agir à distância, como diz Law (1986), é devido a todas essas relações que se montam a seu redor. Quando falamos de múltiplas versões de um fenômeno ancorado em múltiplas versões e visões de mundo, estamos falando de diferentes redes de atores humanos e não humanos, múltiplas realidades que agem para performar as realidades da ciência. Direcionamo-nos também para o conteúdo mesmo dos artigos, que está repleto de relações heterogêneas de atores humanos e não humanos que participam na construção de diferentes versões sobre o TDAH.

\section{Do Conteúdo e dos Nós das Relações nos Artigos}

Ao pensarmos na multiplicidade de versões sobre o TDAH, levando em conta o exposto até aqui, refletimos sobre como esses artigos "falam" do TDAH e que nós de atores humanos e não humanos estão envolvidos nisso. Para isso, voltamos nosso olhar ao conteúdo dos artigos selecionados. Falar de multiplicidade de versões nos faz remeter àquilo que Mol (2007) chama de caráter performativo da realidade, pois "Em lugar de ser vista por uma diversidade de olhos, mantendo-se intocada no centro, a realidade é manipulada por meio de vários instrumentos, no curso de uma série de diferentes práticas" (p. 6).

Temos, nesse universo múltiplo de artigos, aqueles que dizem que o TDAH é o transtorno (sem aspas) mais comum na infância e que ocorre, ou se intensifica, na idade escolar. Já outros, apontam o "transtorno" como uma forma de medicalizar e patologizar os comportamentos infantis. Alguns tratam como uma combinação de fatores biológicos e sociais para o aparecimento e desenvolvimento do TDAH na criança, em outros é um fenômeno complexo e permeado de controvérsias.

Outro exemploé a complexidade do diagnóstico do TDAH, tema constante nos artigos, tratado de diferentes maneiras. O artigo de Caliman (2009) intitulado "A constituição sócio-médica do 'fato TDAH'"dá a tônica quando a autora afirma que os "diagnósticos são constructos cujas fronteiras e limites dependem, em grande parte, da intenção de seus criadores e das pessoas neles enquadradas, mas entre os primeiros e os segundos nem sempre há acordos" (p. 563). O diagnóstico do "transtorno" também é apresentando como multiprofissional e de extrema dificuldade. Muitos artigos apontam que este deve ser feito por vários profissionais em conjunto, além de exames de neuroimagens combinados com ampla experiência profissional, como aponta o estudo de Graeff e Vaz (2008): "É indispensável que o profissional tenha conhecimento clínico de psicopatologia e que possa se valer de outros recursos (como escalas, testes psicológicos e neuropsicológicos)" (p. 342).

Em outros casos, estudos simplesmente utilizam os critérios diagnósticos apresentados pelo DSM como suficientes para o diagnóstico e, como aponta o artigo de Assef, Capovilla e Capovilla (2007), acrescenta um segundo profissional para garantir sua aplicação apurada. Ao definirem seus critérios de inclusão de crianças com TDAH em seu estudo, esses autores afirmam que o "grupo 1 foi formado por 31 crianças com diagnóstico de TDAH, realizado por psiquiatras de dois serviços de psiquiatria infantil do Estado de São Paulo, com base nos critérios da Associação Psiquiátrica Americana - DSM-IV-TRTM" (p. 63-64). O estudo de Freire e Pondé (2005) aponta que "O diagnóstico do TDAH em crianças se baseia em uma história obtida com pais, professores ou outros cuidadores, não requerendo que o médico testemunhe os sintomas ou os obtenha diretamente das crianças" (p. 477). Já o estudo de Rhode et al. (2004) mostra que cabe ao profissional da clínica, de acordo com a história que se apresenta em sua frente, decidir aquilo que não está expresso nos manuais diagnósticos como é o caso da definição do que quer dizer "frequente". Afirmam os autores que "dependendo de onde o clínico colocar o ponto de corte para definir o sintoma como frequente, ele terá mais ou menos indivíduos incluídos na categoria diagnóstica" (p. 124).

Um outro eixo de análise para nossa discussão está nas formas e nas ferramentas utilizadas pelos estudos para montar uma versão de TDAH e traduzi-la para o texto. Aqui optamos por falar mais daqueles artigos nos quais os autores/as foram a campo para traçar seus relatos, os ditos estudos empíricos, sejam eles de caráter quantitativo, qualitativo ou misto. Estes somam 44 artigos, correspondendo a $55 \%$ dos estudos. Observamos que a ação de objetos nesses casos se torna bem evidente. São testes psicológicos, matemáticos, linguísticos, bem como softwares, gráficos, tabelas e estatísticas que participam da construção de determinadas versões de TDAH. Os testes SNAP IV e WISC são os mais utilizados, principalmente para definir amostras na participação das pesquisas e também critérios de inclusão e/ou exclusão em estudos de prevalência e comparativos com grupos controle e grupos clínicos. Ainda que usem muitas vezes os mesmos materiais para a pesquisa, os autores apresentam versões diferentes. Há estudos que mostram um caráter crítico e histórico; já outros se apoiam nas ciências biológicas e em discursos biologizantes; também há os que apresentam uma visão holística de um ser humano indissociável do ambiente em que vive. Todos esses aproximam-se em alguns métodos e distanciam-se nas versões.

Ainda assim, no caso do TDAH, há uma grande disputa em relação à questão das estatísticas com estudos que apontam uma variação muito grande na literatura, principalmente em relação à prevalência, que varia de 3 a $20 \%$ da população. Alguns artigos, como o de Rhode e Hapelbarn (2004), encontram explicação para essa discrepância na variação metodológica dos estudos e das amostras, apontando para um equívoco ou uma confusão dos estudos no modo de identificar o TDAH. Já Brzozowski e Caponi (2012), apontam que a linha divisória entre normal 
e patológico tem se tornado muito tênue pelas práticas em saúde e pela ciência, e que isto funciona como um dispositivo de patologização e medicalização, apontando para um uso pernicioso do dispositivo diagnóstico.

Temos, então, artigos que tratam o TDAH de diferentes maneiras, de acordo com os conceitos de seus respectivos campos. Há trabalhos pautados em um conceito de ciência que se distancia de questões políticas e outros que apontam eventos históricos que apresentam versões que convivem entre si (Mol, 2007). Há artigos que apresentam uma visão de humano sócio-histórico e holístico e outros que se baseiam em descrições biologizantes do corpo como máquina. As/ os autoras/as encontram aportes em objetos distintos, em filiações institucionais e epistemológicas, e cooperam na construção de textos científicos que ajudam e operam na fabricação de diferentes versões acerca do TDAH.

A figura 2 abaixo apresenta as divisões e agrupamentos em núcleos que nos ajudam a visualizar, de uma maneira geral, a forma como cada artigo articula a produção de sua versão.

As categorias descritas na Figura 2 não estavam necessariamente destacadas em todos os artigos e foram pensadas a partir de sentidos que elaboramos no decorrer de todo o processo de pesquisa. Ilustramos esses agrupamentos na Tabela 1.

Observamos que pouco mais da metade dos artigos (42) são estudos empíricos, seguido por artigos teóricos (29) e estudos de caso ou relato de experiência (8). Se utilizarmos como exemplo o número de artigos críticos e o número de artigos comparativos, observamos que a diferença é de apenas um artigo. Ao observarmos os enfoques que confeccionamos, notamos que a maior parte dos artigos (49, divididos entre comparativos, diagnósticos, farmacológicos, biológicos e descritivos) está comprometida em produzir uma versão do TDAH que se articula, com maior ou menor ênfase, na biologicidade do "transtorno", ainda que, como vimos, essa base seja imprecisa. Também observamos que a maior parte dos textos parte da ideia de que o transtorno existe enquanto uma realidade. Por outro lado, alguns se articulam enquanto uma resistência a essa versão de TDAH e cooperam na sustentação da ideia de que o transtorno existe enquanto uma prática social. Isso está relacionado com os agenciamentos e movimentos que participam na fabricação de realidades.

Sinteticamente, em determinados lugares, o TDAH é um conjunto de agentes que podem incluir exames de neuroimagem, intervenções multiprofissionais, laboratórios, máquinas, tecnologia ou "apenas" a opinião e a experiência clínica de um profissional. Também pode ser um discurso de patologização de comportamentos e um silenciamento de subjetividades.

\section{Das Redes dos Artigos II: Para um Olhar mais Espcífico sobre a Multiplicidade}

Apresentaremos nesta seção três textos selecionados que ilustram três versões do TDAH. Selecionamos os seguintes artigos: (1) "A constituição sócio-médica do "fato TDAH'", de Luciana Vieria Caliman (2009); (2) “Contribuição dos fatores de risco psicossociais para o Transtorno de Déficit de Atenção/Hiperatividade", de Marcio M. Vasconcelos, Ana Flávia de A. Malheiros, Jairo Werner Jr., Adriana R. Brito, Jane Bardawil Barbosa, Ítalo S. Oliveira Santos e Daniel Fampa N. Lima (2005); (3) "Transtorno de déficit de atenção/hiperatividade: atualização", de Luis Augusto Rohde e Ricardo Halpern (2004). Estes estudos contam com

Tipos de Estudos

- Teóricos $\quad$ - Empíricos $\left\{\begin{array}{l}\text { Quantitativos } \\ \text { Qualitativos } \\ \text { Mistos }\end{array}\right.$

Enfoques

\begin{tabular}{|c|}
\hline Biológico \\
Foco principal é afirmar ou \\
então investigar as raízes \\
biológicas do TDAH com \\
base nos pressupostos da \\
ciência moderna.
\end{tabular}

Explicativo

Em certa medida, tomam o TDAH como uma junção de fatores biológicos e sociais explicando essa relação e seus efeitos na vida de crianças diagnosticadas.

\begin{tabular}{|c|}
\hline $\begin{array}{c}\text { Farmacológico } \\
\text { Atenção particular à } \\
\text { farmacologia do "transtorno" } \\
\text { em termos de medicamentos e } \\
\text { sua ação no corpo. }\end{array}$ \\
\hline $\begin{array}{c}\text { Comparativo } \\
\text { Maioria estudos empíricos } \\
\text { que comparam grupos clínicos } \\
\text { e não clínicos acerca do } \\
\text { TDAH e suas nuances, } \\
\text { geralmente ancorados no } \\
\text { método científico moderno. }\end{array}$ \\
\hline
\end{tabular}

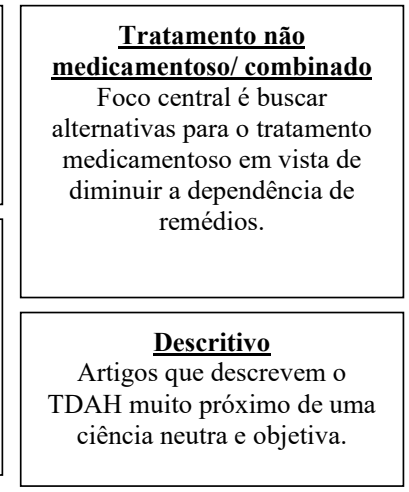

Figura 2. Organização em núcleos e de acordo com o enfoque. 
Tabela 1

Quantidade de artigos por enfoque e por tipo de estudo

\begin{tabular}{|c|c|c|c|c|c|c|}
\hline \multirow[b]{2}{*}{ Enfoque } & \multicolumn{6}{|c|}{ Tipos de estudos } \\
\hline & Empíricos quanti & Empíricos quali & Empíricos mistos & Teóricos & $\begin{array}{l}\text { Relatos de exper., } \\
\text { estudos de casos e outros }\end{array}$ & $\begin{array}{c}\text { Total de estudos } \\
\text { por enfoque }\end{array}$ \\
\hline Comparativo & 16 & & & & & 16 \\
\hline Diagnóstico & 1 & 1 & 4 & 3 & 3 & 12 \\
\hline Descritivo & 3 & 3 & 4 & 4 & & 14 \\
\hline Biológico & 1 & & & 3 & & 4 \\
\hline Farmacológico & 2 & & 1 & & & 3 \\
\hline Crítico & & 4 & & 10 & 3 & 17 \\
\hline Explicativo & & & 1 & 6 & 1 & 8 \\
\hline $\begin{array}{l}\text { Tratamento não } \\
\text { medicamentoso/ } \\
\text { combinado }\end{array}$ & & 1 & 1 & 3 & 1 & 6 \\
\hline Total & 23 & 9 & 11 & 29 & 8 & 80 \\
\hline
\end{tabular}

um grande número de citações, 32 para o texto (1), 38 para o (2) e 183 para o (3), ou seja, tem um impacto grande em termos de alcance e ação à distância na produção científica brasileira acerca do TDAH.

\section{O TDAH no Artigo (I), de Luciana Vieria Caliman}

O artigo (1), de autoria de Luciana Vieira Caliman, foi publicado em 2009 na revista Psicologia \& Sociedade, da Associação Brasileira de Psicologia Social (ABRAPSO), avaliada no estrato $\mathrm{A} 2$ pela CAPES na área de psicologia. De acordo com nosso levantamento, conta com 32 citações nos dois motores de busca que utilizamos (Google Acadêmico e Scopus). Caliman é pós-doutora em psicologia e professora da área na Universidade Federal do Espírito Santo (UFES). Em seu Lattes, encontramos 17 artigos publicados - seja com colaboradores/as ou de única autoria -, dentre os quais 12 se referem de alguma forma ao TDAH e suas nuances. A autora desenvolve projetos de pesquisa que discutem, de modo geral, a relação do diagnóstico neurocientífico na sociedade e as políticas públicas de saúde e educação. $\mathrm{O}$ artigo (1), especificamente, trata-se de um estudo teórico com um enfoque na contextualização da construção do TDAH enquanto um "fato", considerando diversos aspectos desta "realidade". De acordo com a autora, o "TDAH é analisado como um objeto empírico e social criado na constelação política, econômica e científica característica da segunda metade do século XX" (Caliman, 2009, p. 136).

Em sua trajetória como pesquisadora, é possível identificar desde o seu primeiro projeto relacionado ao diagnóstico neurocientífico um posicionamento crítico com relação aos efeitos das chamadas ciências duras na construção de modos de vida social. A autora entende que as ciências sociais têm o papel de fazer um contraponto ao discurso médico-científico, de modo a identificar suas contribuições e principalmente as reduções que pode promover. Vê-se, então, que seu compromisso científicopolítico localiza-se na produção de um contraponto àquilo que a autora entende como um prejuízo social do discurso diagnóstico neurocientífico, da política pública nele sustentada e da medicalização.

No artigo que analisamos, Caliman (2009) aborda o TDAH como um fenômeno que se constitui por diversas relações, histórias de política, moral e ciência que estão emaranhadas, mas que de alguma forma são "ocultadas" na versão neurobiológica do "transtorno". Para isso, utiliza o argumento de LudwickFleck sobre os diversos níveis da constituição de um fato científico. Para Caliman (2009), "Em suas bases, encontramos vozes destoantes, opiniões e escolhas pessoais e contingências institucionais. Em sua última face, ele é transformado em uma realidade neutra e objetiva, imune aos conflitos e direções que, na verdade, fizeram parte de sua constituição" (p. 135).

Nesse sentido, a autora aponta que a interpretação neurobiológica revela apenas o nível aparente do TDAH e sua análise busca demonstrar como aquilo que está nos níveis menos superficiais da constituição do "transtorno" enquanto um fato participa da ação. A autora foca, em sua pesquisa, a construção da versão neurobiológica do "transtorno" e como e por que essa versão e as práticas dela decorrentes são apresentadas como uma realidade única.

Traçando um histórico da psiquiatria enquanto discurso biológico e da construção do DSM, o artigo nos apresenta argumentos que apontam a interpretação biológica como central na fabricação de novas discriminações sociais. A filosofia do risco, os exames de neuroimagens e a tomada da atenção enquanto fator importante na vida das pessoas alimentaram estas discriminações, voltando o olhar da ciência para as nuances do "transtorno".

A autora retoma estudos da década de 1990, que traziam como argumento central a filosofia do risco em termos de comprometimento social e econômico das pessoas (Caliman, 
2009). Produz uma versão do transtorno que não se sustenta enquanto uma verdade única, pois para a autora, é carregada de diversos aspectos que ficam silenciados na interpretação neurobiológica. Para a autora, dois argumentos são chaves para tal silenciamento na busca da realidade objetiva do "transtorno". O primeiro (1) é de que causa perigos e danos para as pessoas. Esse argumento ancora-se na filosofia do risco e sustenta a busca por provas cientificas dos prejuízos na vida das pessoas. Porém, para Caliman (2009), "A decisão sobre o que é uma consequência adversa e sobre a intensidade na qual ela se torna patológica depende do que uma sociedade acredita ser bom ou ruim, certo ou errado" (p.141). Nesse sentido, por ameaçar a qualidade de vida das pessoas, em termos econômicos, sociais e morais específicos, baseados na filosofia do risco, a interpretação neurobiológica do TDAH ganha força ou, em outras palavras, aliados. O segundo argumento (2) afirma que o transtorno pode ser visualmente identificado. Este argumento ancora-se no uso dos exames de imagem cerebral, pesquisas genéticas e moleculares, que reiteram o "transtorno" como "real porque é visível e biológico" (Caliman, 2009. p. 141).

A autora aponta que a busca pela realidade do transtorno está ligada à crença na objetividade e neutralidade da psiquiatria biológica que foi se desenhando a partir da década de 1970. Caliman (2009) apresenta, então, uma crítica a esse nível superficial do conhecimento acerca do transtorno, que credita aos riscos e às evidencias biológicas a sua realidade enquanto um fato científico. O TDAH de que fala a autora é performado por muito mais elementos que a interpretação neurobiológica apresenta: "não se resumiu, e não se resume, à interrogação sobre sua natureza real e biológica. Ele é formado por outras questões de caráter político, ético, econômico e moral que dialogam com as exigências da economia da atenção atual" (Caliman, 2009. p. 143). Para essa versão existir, é preciso engendrar-se em outros objetos, na economia política e nos níveis mais profundos da constituição do "transtorno". Utiliza estudos sobre a história das ciências e especificamente sobre a psiquiatria, aportes discursivos da filosofia do risco e de questionamentos dos postulados de neutralidade e objetividade científica.

\section{O TDAH no Artigo (2),}

\section{de Marcio Vasconcelos et al.}

O artigo (2), de Vasconcelos et al. (2005), conta com 38 citações e foi publicado na revista Arquivos de Neuropsiquiatria, pela qual a Academia Brasileira de Neurologia é responsável. É avaliada no estrato B2 pela CAPES na área de medicina II e A2 na área de psicologia. Vasconcelos é professor de pediatria e neurologia da Universidade Federal Fluminense e possui doutorado em neurologia pela mesma instituição. De seus 22 artigos publicados em periódicos, 3 são a respeito de TDAH. Trata-se de um estudo empírico de método quantitativo e qualitativo, que aponta o TDAH como um transtorno neurobiológico com fatores psicossociais que atuam correlacionados. Nas palavras dos/as autores/as, o objetivo é "investigar a correlação dos fatores de risco psicossociais com o diagnóstico de TDAH" (Vasconcelos et al., 2005, p. 69).

Um olhar para a trajetória de Vasconcelos como pesquisador permite identificar que o texto discutido aqui é fruto de seu trabalho de mestrado (Transtorno de Déficit de Atenção/Hiperatividade: Prevalência e Contribuição dos Fatores Psicossociais), orientado por Jairo Werner Júnior (coautor do artigo). Ambos os autores se localizam no campo da psiquiatria infantil e apresentam em suas trajetórias tanto uma preocupação com os fatores médico-biológicos e diagnósticos (como genética e exames de neuroimagem) quanto com uma leitura dos aspectos psicossociais do desenvolvimento infantil, recorrendo a autores para além do campo médico como, por exemplo, Lev Vygotsky.

A compreensão desses aspectos da trajetória do autor (autores) sinaliza para um engajamento científicopolítico misto que busca compreender o TDAH a partir de uma linguagem familiar às neurociências (prevalência, diagnóstico, neuroimagem), mas também considerando fatores psicológicos e sociais. No artigo (2), de Vasconcelos et al. (2005), o foco não são as raízes mais profundas da constituição de um transtorno, mas a afirmação de um discurso da realidade biológica do TDAH com a adição de fatores, objetos e relações próprios do campo social, que desencadeiam no aparato biológico sua manutenção e manifestação. Segundo o texto "A influência dos fatores genéticos na etiologia do TDAH tem sido intensamente explorada. Há consenso de que a contribuição genética é substancial e acarreta vulnerabilidade ao TDAH, provavelmente interagindo com fatores ambientais" (Vasconcelos et al., 2005, p. 69). O estudo contou com 101 crianças, que compareceram divididas em três grupos e comparadas entre si. De acordo com os critérios de seus autores, "O diagnóstico de TDAH foi confirmado em 69 crianças $(17,1 \%)$, Foi possível parear 45 dos 69 alunos diagnosticados. Entretanto, apenas 31 alunos controles $(58,9 \%)$ compareceram para o atendimento e foram incluídos na pesquisa, gerando 31 pares de casos e controles" (p. 69).

Os critérios utilizados para a definição diagnóstica das crianças que participaram da pesquisa foram apresentados em artigo anterior dos mesmos autores/as, um estudo de prevalência em uma escola primária no Rio de Janeiro. A inclusão foi baseada nos critérios diagnósticos do DSM-IV em quesitos apresentados a pais e professores, em que o enquadre em seis de nove quesitos aponta para a presença do "transtorno". Assim, nos apresentam uma versão de TDAH que se constrói enquanto uma realidade biológica da qual participam também o DSM-IV, pais, professores, grupos controle e de casos, sintomas, questionários e respostas. Entretanto, participam também os fatores psicossociais, alvos da pesquisa do artigo (2), que foram trazidos em cena por meio de análises estatísticas de uma gama de 
questionários apresentados aos cuidadores das crianças envolvidas no estudo. No caso do artigo (2), a estatística foi utilizada para definir padrões de fatores psicossociais e aqueles que se mostraram relevantes para os autores foram: "brigas conjugais no passado, separação dos pais e depressão na mãe" (Vasconcelos et al., 2005, p. 72).

Nesse sentido, para os autores, estes seriam fatores que contribuem para a manutenção e desencadeamento do TDAH. Estamos falando de uma versão que corrobora as interpretações neurobiológicas do "transtorno" e adicionam a esta a contribuição de outros atores humanos e não humanos como desencadeadores ou mantenedores. O artigo de Vasconcelos et al. (2005) não fala de ciência enquanto discurso, ou de transtorno enquanto constructo com diversas contingências, não questionando também de onde vem a realidade do TDAH. Fala-se de uma versão de fatores psicossociais que desencadeiam ou fazem agir as marcas biológicas do corpo, utilizando questionários, critérios diagnósticos, entrevistas com pais e professores, tabelas e médias estatísticas.

\section{O TDAH no Artigo (3), de Rohde e Halpern}

O artigo (3), de autoria de Rohde e Halpern, foi publicado em 2004 no periódico Jornal de Pediatria, publicação da Sociedade Brasileira de Pediatria, classificada no estrato B2 pela CAPES na área de seus autores, medicina II, e B1 na área de Psicologia (qualis 2013). Conta com um total de 183 citações e é a única publicação sobre o tema de Halpern, que é professor da Universidade Federal de Ciências Médicas de Porto Alegre, atuando na área de pediatria do desenvolvimento e comportamento. Rohde é professor de Psiquiatria e Medicina Legal da Universidade Federal do Rio Grande do Sul, diretor do Programa de Déficit de Atenção/Hiperatividade no Hospital de Clínicas de Porto Alegre e membro do grupo para Transtornos Disruptivos do Comportamento e TDAH do DSM-5. Além disso, possui doutorado e livre docência em ciências médicas. O artigo (3) é uma entre as 83 publicações sobre TDAH deste autor, sendo quatro em português e 79 em língua estrangeira, de um total de 244 publicações em periódicos. Ele também coordena 26 projetos de pesquisa relacionados ao TDAH, com o primeiro iniciado em 1996. A partir da análise do texto e da trajetória de seus autores, identificamos a preocupação científica e política de afirmar as bases biológicas do transtorno e a precisão de seu diagnóstico.

Trata-se de uma "atualização" acerca do TDAH, em que os autores definem o público-alvo de seu estudo já no resumo do trabalho em termos de objetivo: "o presente estudo busca uma revisão atual, para o pediatra clínico, dos principais aspectos do histórico, epidemiologia, etiologia, neurobiologia, quadro clínico, comorbidades, diagnóstico, evolução e tratamento do transtorno" (Rohde \& Halpern, 2004). Aqui também jaz definida pelos autores a gama de matérias que utilizam para sua versão de TDAH. Centrado em pesquisas genéticas e estudos de prevalência, o artigo traz o "transtorno" enquanto uma realidade biológica centrada nas evidências das imagens cerebrais e de estudos genéticos. Podemos dizer que se encontra situado naquilo que Caliman (2009) chamou de substrato mais superficial na construção do TDAH enquanto um fato, ainda que coloquem em cena dois parágrafos acerca de fatores psicossociais, que nas palavras dos autores, apenas parecem ter relação com o TDAH.

Assim como Caliman (2009), fazem um histórico sobre a realidade do transtorno e como suas nomenclaturas foram mudando ao longo do tempo. Porém, no artigo (3), essas mudanças decorrem não de fatores políticos, morais ou sociais, mas de um avanço nas pesquisas de base genética e neuroquímica: "Na década de 40, surgiu a designação 'lesão cerebral mínima', que, já em 1962, foi modificada para 'disfunção cerebral mínima', reconhecendo-se que as alterações características da síndrome relacionam-se mais a disfunções em vias nervosas do que propriamente a lesões nas mesmas" (Rohde \& Halpern, 2004, p. 61).

Em meio a referências a estudos de prevalência, de aceitação da condição biológica do TDAH, dicas para um diagnóstico preciso, os autores colocam em cena uma preocupação com um diagnóstico objetivo e exato dignos da realidade biológica do TDAH: "a desatenção, a hiperatividade ou a impulsividade como sintomas isolados podem resultar de muitos problemas na vida de relação das crianças [...] ou podem estar associados a outros transtornos comumente encontrados na infância e adolescência"(Rohde \& Halpern, 2004, p. S64). Em certa medida, essa preocupação tem a ver com a produção de um endosso da realidade neurobiológica do TDAH e também na construção de uma confiança na psiquiatria biológica e nos exames de imagens e estudos genéticos. A versão do TDAH do artigo (3) é uma versão centrada na objetividade e neutralidade das evidências científicas. Assim, os autores em seu artigo falam de uma versão de TDAH que existe por meio da ciência, das pesquisas genéticas e de neuroimagens, do medicamento e de um diagnóstico da realidade biológica do "transtorno".

\section{Sobre Diferentes Agenciamentos}

Cada artigo possui uma configuração específica que desenha o TDAH em conformidade com diferentes alinhamentos científico-políticos. É possível que pareça que estamos lidando com diferentes visões de um único objeto (TDAH), uma pluralidade de visões que se ancoram nas filiações de áreas, institucionais e objetos utilizados para se falar do "transtorno" de acordo com cada perspectiva. Buscamos, porém, falar de múltiplas realidades acerca do TDAH, ou seja, não de como estes autores observam, mas de como fazem diferentes TDAHs. Falar de realidades múltiplas é dizer de diferentes versões de objetos que podem ou não estar relacionados e que as diferentes materialidades ajudam a performar diferentes práticas (Mol, 2007). 
Nesse sentido, se estamos falando de versões, de múltiplas realidades, estamos buscando como estas se conectam, como se distanciam e o que agenciam. Estas três versões diferentes sobre o TDAH se conectam, se distanciam e se sobrepõem. Cada texto agencia o "transtorno" de diferentes maneiras, recorrendo a diferentes associações. Para Vasconcelos et al. (2005), os critérios diagnósticos do DSM-IV em formas de perguntas para pais e professores podem incluir crianças em estudo, colocando-as como portadoras de TDAH. Para Rohde e Halpern (2004), apenas uma criteriosa avaliação com o auxílio de estudos genéticos e de imagens pode dar conta de tal tarefa. Já para Caliman (2009), o TDAH deve ser analisado enquanto um constructo com diversas contingências políticas sociais e morais a serem levadas em consideração.

Ao trazermos em cena as sagazes análises de Mol (2007), percebemos que estas versões são performadas de maneiras diferentes em termos de materiais, procedimentos e atributos. A versão de TDAH de Caliman (2009), no artigo (1), fala de controvérsias e complexidades do "transtorno" (atributos). A autora o historiciza (procedimento), colocando em cena textos da filosofia das ciências, da sociologia, de releituras dos elementos da psiquiatria biológica, moral, política e cultura (materiais). Vasconcelos et al. (2005) apontam um TDAH como uma realidade biológica atravessada por alguns fatores psicossociais (atributos), fazendo uma investigação em campo padronizada por medidas estatísticas (procedimento) por meio de testes, questionários, softwares de análise, critérios diagnósticos, tabelas e gráficos (materiais). Por fim, o artigo (3) de Rohde e Halpern (2004) performa um TDAH predominantemente biológico e cerebral (atributos). Isso é feito por meio da retomada de estudos biológicos e estatísticos (procedimento) ao colocarem em cena genes, moléculas, medicamentos, neuroimagens, critérios diagnósticos, estudos de prevalência e laboratórios (materiais). Esse conjunto (materiais, procedimento e atributo) performa, por meio de seus agenciamentos, cada versão do TDAH.

As categorias diagnósticas do TDAH, por exemplo, aproximam os textos, ainda que estes construam diferentes versões sobre o mesmo a partir delas. O boom farmacológico e o avanço tecnológico das pesquisas e das imagens cerebrais não impediram que textos ou pesquisas que negassem ou questionassem a realidade biológica do TDAH coexistissem. Porém, se objetos com um mesmo nome coexistem, eles se sobrepõem na prática, um sendo mais privilegiado que o outro (Mol, 2002). Ao observarmos, por exemplo, o número de citações (183) do artigo de Rohde e Halpern (2004), estas nos apontam para a "força" de seu texto e seu impacto na produção científica e nas práticas. Assim, ele age à distância, produzindo uma rede científica específica potente, no sentido de que alcança muito mais aliados (Law, 1986). A noção de ciência neutra e objetiva da psiquiatria biológica foi e ainda é muito privilegiada desde a década de 1980 .

\section{CONSIDERAÇÕES FINAIS}

Embora coexistam diferentes versões de TDAH, existem aquelas que se sobrepõem e prevalecem em um movimento dinâmico. Vimos que aquelas que se conectam com a tarefa de produzir uma única descrição biológica do TDAH se sobrepõem àquelas que, por exemplo, falam que o "transtorno" deveria ser pensado de acordo com todos os seus agenciamentos humanos e não humanos. Na medida em que essa coexistência de versões se dá em um mundo ainda dominado por uma descrição de realidade enquanto única, independente e estável, faz-se necessário hierarquizar as versões e minimizar os efeitos disruptivos de suas diferenças.

Buscamos identificar e analisar versões que gerem efeitos diferentes daqueles gerados pelo saber ancorado na neutralidade, objetividade e generalização. As premissas de uma ciência moderna, biologicista e desengajada de questões políticas geram múltiplos efeitos, mas preocupa-nos os efeitos de medicalização e patologização das relações que produzem corpos uniformes voltados a uma lógica produtiva e de consumo. Estabelecem também a ciência enquanto um dispositivo de verdade única e de um saber melhor sobre a vida. Como vimos, parte da literatura corrobora essa produção, contudo, outra parte busca diferentes associações e algumas são justamente críticas a essas premissas.

Produzir uma visão sobre a multiplicidade do TDAH e ao mesmo tempo sobre o processo científico que opera em uma rede de produções de diferentes versões é, então, uma estratégia implosiva. Esperamos ter o efeito de abrir o campo para a emergência de novas versões e/ou fortalecer versões já existentes, podendo produzir outras (melhores, esperamos) reverberações sociais. 


\section{REFERÊNCIAS}

Anvisa. (2013). Boletim de Fármacoepidemiologia do SNGPC, 2(2).

Assef, E. C. S., Capovilla, A.G.S., \& Capovilla, F. C. (2007). Avaliação do controle inibitório em TDAH por meio do Teste de Geração Semântica. Psicologia: Teoria e Prática, 9(1), 6174. Recuperado de http://editorarevistas.mackenzie.br/index. php/ptp/article/viewFile/680/399

Brzozowski, F. S., \& Caponi, S. (2012). Determinismo biológico e as neurociências no caso do transtorno de déficit de atenção com hiperatividade. Physis, 22(3), 941-961.

Caliman, L. V. (2009). A constituição sócio-médica do "fato TDAH”. Psicologia \& Sociedade, 21(1), 135-144. Recuperado de http://www.scielo.br/pdf/psoc/v21n1/16.pdf

Ferreira, R. R. (2015). A medicalização nas relações saber-poder: Um olhar acerca da infância medicalizada. Psicologia em Estudo, 20(4), 587-598.

Freire, A. C. C., \& Pondé, M. P. (2005). Estudo piloto da prevalência do transtorno de déficit de atenção e hiperatividade entre crianças escolares na cidade do Salvador, Bahia, Brasil. Arquivos de Neuropsiquiatria, 63(2b), 474-478. Recuperado de http://www.scielo.br/scielo.php?script=sci_arttext\&pid=S 0004282X2005000300020\&lng $=$ en\&tlng $=$ pt

Gomes, M., Palmini A., Barbirato F., Rohde, L. A., \& Mattos, P. (2007). Conhecimento sobre o transtorno do déficit de atenção/ hiperatividade no Brasil. Jornal Brasileiro de Psiquiatria, 56(2) 94-101. Recuperado de http://www.scielo.br/scielo.php?pid= S004720852007000200004\&script $=$ sci_arttext

Graeff, R. L., \& Vaz, C. E. (2008). Avaliação e diagnóstico do transtorno de déficit de atenção e hiperatividade (TDAH).
Psicologia USP, 19(3), 341-361. Recuperado de http://www. redalyc.org/articulo.oa?id=305123728005

Latour, B. (2011). Ciência em ação: Como seguir cientistas e engenheiros sociedade afora. São Paulo: Editora Unesp.

Law, J. (1986). The heterogeneity of texts. In M. Callon, J. Law, \& A. Rip (Orgs.). Mapping the dynamics of science and technology (pp. 67-83) London: Macmillan Press.

Mol, A. (2002). The body multiple: Ontology in medical practice. Durham, NC: Duke University.

Mol, A. (2007). Política ontológica: Algumas ideias e várias perguntas. In J. A. Nunes, \& R. Roque (Org.), Objectos impuros: Experiências em estudos sociais da ciência. Porto: Edições Afrontamento.

Moraes, M. O. (2001/2002). Sobre a noção de rede e a singularidade das ciências. Revista Documenta, 8(12/13), 57-70.

Moraes, M. O. (2004). A ciência como rede atores: Ressonâncias filosóficas. História, Ciencias, Saúde - Manguinhos, 11(2), 321-333.

Rohde L. A., \& Halpern, R. (2004). Transtorno de déficit de atenção/hiperatividade: Atualização. Jornal de Pediatria, 80(2), 61-70.

Vasconcelos, M. M., Malheiros, A. F A., Werner Jr., J., Brito, A. R., Barbosa, J. B., Santos, Í. S. O., \& Lima, D. F. N. (2005). Contribuição dos fatores de risco psicossociais para o transtorno de déficit de atenção/hiperatividade. Arquivos de Neuropsiquiatria, 63(1), 68-74. Recuperado de http://www. scielo.br/scielo.php?script $=$ sci_arttext\&pid=S0004-282X200 5000100013\&lng=en\&tlng $=\mathrm{pt}$ 